\title{
Integrated microarray analysis of key genes and a miRNA-mRNA regulatory network of early-onset preeclampsia
}

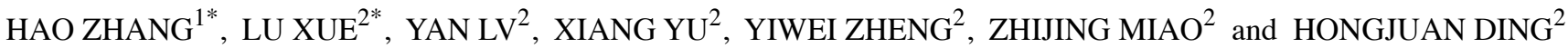 \\ ${ }^{1}$ Department of Internal Medicine, The Affiliated Drum Tower Hospital of Nanjing University Medical School, \\ Nanjing, Jiangsu 210008; ${ }^{2}$ Department of Obstetrics and Gynecology, Women's Hospital of Nanjing Medical University, \\ Nanjing Maternity and Child Health Care Hospital, Nanjing, Jiangsu 210004, P.R. China
}

Received April 3, 2020; Accepted August 18, 2020

DOI: $10.3892 / \mathrm{mmr} .2020 .11551$

\begin{abstract}
Early-onset preeclampsia (EOPE) is a serious threat to maternal and foetal health. The present study aimed to identify potential biomarkers and targets for the treatment of EOPE. Expression profiles of placenta from patients with EOPE and healthy controls (GSE103542, GSE74341 and GSE44711) were downloaded from the Gene Expression Omnibus database. Integrated analysis revealed 246 genes and 28 microRNAs (miRNAs) that were differentially expressed between patients with EOPE and healthy controls. Differentially expressed genes (DEGs) were primarily enriched in 'biological processes', such as 'cell adhesion', 'female pregnancy', 'extracellular matrix organization' and 'response to hypoxia'. Significant pathways associated with DEGs primarily included 'focal adhesion', 'ECM-receptor interaction', 'PI3K-Akt signaling' and 'ovarian steroidogenesis'. A Protein-Protein Interaction network of DEGs was constructed using the Search Tool for the Retrieval of Interacting Genes/Proteins online database, and epidermal growth factor receptor, collagen $\alpha-1(\mathrm{I})$ chain, secreted phosphoprotein 1 , leptin (LEP), collagen $\alpha-2$ (I) chain (COL1A2), plasminogen activator inhibitor 1 (SERPINE1), Thy-1 membrane glycoprotein, bone morphogenetic protein 4, vascular cell adhesion protein 1 and matrix metallopeptidase 1 were identified as hub genes. The alterations of hsa-miR-937, hsa-miR-148b*, hsa-miR-3907, hsa-miR-367*, COL1A2, LEP and SERPINE1 in placenta were validated using our local samples. Our research showed that the expression of
\end{abstract}

Correspondence to: Dr Zhijing Miao or Dr Hongjuan Ding, Department of Obstetrics and Gynecology, Women's Hospital of Nanjing Medical University, Nanjing Maternity and Child Health Care Hospital, 123 Mochou Road, Nanjing, Jiangsu 210004, P.R. China

E-mail: 854422290@qq.com

E-mail: njdinghj@163.com

*Contributed equally

Key words: early onset preeclampsia, Gene Expression Omnibus, integrated bioinformatics analysis
hsa-miR-937, hsa-miR-1486*, hsa-miR-3907, hsa-miR-367* and hub genes in the placenta were closely associated with the pathophysiology of EOPE. hsa-miR-937, hsa-miR-1486*, hsa-miR-3907, hsa-miR-367* and hub genes could serve as biomarkers for diagnosis and as potential targets for the treatment of EOPE.

\section{Introduction}

Preeclampsia (PE), characterized by hypertension and proteinuria, is a pregnancy-specific disease that can cause foetal intrauterine growth restriction and premature birth (1). PE can be accompanied by eclampsia, uncontrolled hypertension or systemic inflammation (2) and has become the leading cause of maternal mortality in the United States (3). PE is closely associated with genetic background, and the only cure for PE is delivering the foetus and placenta (4). Early-onset $\mathrm{PE}$ (EOPE) is PE diagnosed before 34 weeks of gestation (5). Compared with late-onset PE (LOPE), EOPE has a higher mortality rate (6) and more often leads to foetal growth restriction (7).

MicroRNAs (miRNAs/miRs) are small non-coding single-stranded RNAs that play important roles in a number of diseases, such as malignant tumours (8) and cardiovascular diseases (9). miRNAs can bind to target genes and thereby inhibit the translation of target genes or promote the degradation of mRNAs. The analysis of miRNA-mRNA regulatory networks has provided novel insights into the pathogenesis of various diseases. For instance, Wang et al (10) discovered that miR-223-3p can relieve spinal cord injury via negatively regulating the expression of receptor-interacting protein 3 .

Microarray technology is useful for detecting the expression of genes and non-coding RNAs in a high-throughput manner (11). Microarray technology has been widely employed in studying transcriptional alterations in various diseases. For example, Huang et al (12) studied traumatic brain injury-related genes with gene expression microarray analysis. Tan et al (13) utilized a miRNA expression microarray assay to identify miR-302b-3p as regulator of skin fibroblast senescence.

Given the large amounts of data generated by microarray experiments, integrated analyses yield information of greater confidence and content than each experiment individually (14). In the current study, a comprehensive strategy was 
employed to analyse gene and miRNA expression microarray data concerning EOPE. The aim of the present study was to discover novel biomarkers for EOPE diagnosis and new targets for EOPE treatment, and to reveal a potential miRNA-mRNA regulatory network contributing to the pathogenesis of EOPE.

\section{Materials and methods}

Microarray data collection. 'Early onset preeclampsia' was used as key word to search the Gene Expression Omnibus (GEO, https://www.ncbi.nlm.nih.gov/geo/) database (15). The inclusion criterion for a GEO series was the inclusion of microarray data of placenta tissues from patients with EOPE and healthy controls. Normalized expression matrixes of GSE103542, GSE74341 and GSE44711 (presented in Table I) were downloaded for further study.

Identification of differentially expressed genes (DEGs). The expression matrixes of GSE74341 and GSE44711 were annotated with official gene symbols and then merged by common gene symbols. RankProd (v3.12.0) (16) is an R package that employs the rank product method to identify DEGs with application in microarray data meta-analysis. The $\mathrm{R}$ package metaMA (v3.1.2) (17) combines either P-values or modified effect sizes across different platforms to identify DEGs. The merged expression matrix was entered into each of the RankProd and metaMA pipelines. The criteria for identifying a gene as a DEG was I[fold-change (FC)] $>2$ and percentage of false prediction ( $\mathrm{pfp}$ ) $<0.05$ (RankProd method) or false discovery rate (FDR) $<0.05$ (metaMA method). A Venn diagram of DEGs identified by RankProd and metaMA was then plotted with using InteractiVenn (http://www.interactivenn.net/) (18). Genes identified as DEGs by both RankProd and metaMA were deemed DEGs for the purposes of the present study. The clustering heat maps of DEGs were plotted using the pheatmap R package (v1.0.12) (19).

Functional annotation of DEGs. The online Database for Annotation, Visualization and Integrated Discovery (DAVID, v6.8, https://david.ncifcrf.gov/) $(20,21)$ was used for Gene Ontology (GO) $(22,23)$ and Kyoto Encyclopedia of Genes and Genomes (KEGG) (24) pathway enrichment analyses of the DEGs.

Construction of a protein-protein interaction (PPI) network. The list of DEGs was uploaded to the Search Tool for the Retrieval of Interacting Genes/Proteins (STRING, v11.0, https://string-db.org/) (25) database to construct the PPI network with the minimum required interaction score set as medium confidence (0.400). The PPI network was visualized and analysed using Cytoscape (v3.7.1) (26), and important gene modules were identified with the Cytoscape plugin MCODE (27).

Identification of differentially expressed miRNAs (DEMs). The expression matrix of GSE103542 was processed with the R package limma (v3.44.3) (28), according to the developer's manual. DEMs were defined according to $\mid \log 2 \mathrm{FCl}>2$ and $\mathrm{P}<0.05$. A volcano plot of the $\log _{2}(\mathrm{FC})$ and $\mathrm{P}$-value of miRNAs was plotted using the ggplot2 R package (v3.3.2) (29).
Construction of a DEM-target DEG connection network. Target genes of DEMs were predicted using the miRNAtap R package (v1.22.0) (30), which integrates data from the PicTar, DIANA, TargetScan, miRanda and miRDB databases as previously described (31). Common genes between upregulated DEGs and target genes of downregulated DEMs were deemed predicted target DEGs of downregulated DEMs. Similarly, overlapping genes between downregulated DEGs and target genes of upregulated DEMs were deemed predicted target DEGs of upregulated DEMs. The DEM-target DEG network was constructed using the software Cytoscape (v3.7.1).

Sample collection. All samples were collected from pregnant women who gave birth in The Women's Hospital of Nanjing Medical University from January 2019 to December 2019. The criteria for EOPE designation were hypertension (blood pressure ( $>140 / 90 \mathrm{mmHg}$ ), proteinuria ( $\geq 300 \mathrm{mg} / \mathrm{d}$ ) and being diagnosed prior to 34 weeks of gestation $(1,32)$. The exclusion criteria included diabetes, gestational diabetes, pre-existing hypertension, congenital anomalies, infection and alcohol/drug use. Healthy matched women with no complications were recruited as controls. Clinical information of patients with EOPE and healthy controls is listed in Table II. All women participating in the study signed informed consent for the collection of placenta tissues and clinical information. This research was approved by Human Research Ethics Committee of The Women's Hospital of Nanjing Medical University (Nanjing, China; approval no. KY-024).

Reverse transcription-quantitative polymerase chain reaction $(R T-q P C R)$. Placenta tissues were dissolved in TRIzol ${ }^{\circledR}$ reagent (cat. no. 93289; Sigma-Aldrich; Merck KGaA), and then RNA was extracted using the RNAprep pure Tissue Kit (cat. no. DP431; Tiangen Biotech Co., Ltd.) according to the manufacturer's instructions. Reverse transcription was performed using a PrimeScript ${ }^{\mathrm{TM}}$ RT Reagent Kit with gDNA Eraser (cat. no. RR047A; Takara Bio, Inc.), according to the manufacturer's instructions. gDNA was removed by incubating the samples at $42^{\circ} \mathrm{C}$ for 2 min; cDNA was then synthesized at $37^{\circ} \mathrm{C}$ for $15 \mathrm{~min}$ and $85^{\circ} \mathrm{C}$ for $5 \mathrm{sec}$. RT-qPCR of the DEGs was conducted using a CellAmp ${ }^{\mathrm{TM}}$ Direct TB Green ${ }^{\circledR}$ RT-qPCR Kit (cat. no. 3735A; Takara Bio, Inc.) and an ABI ViiA ${ }^{\mathrm{TM}} 7$ System (cat. no. 4453536; Thermo Fisher Scientific, Inc.). The thermocycling conditions consisted of an initial denaturation at $95^{\circ} \mathrm{C}$ for $30 \mathrm{sec}$, followed by 40 cycles at $95^{\circ} \mathrm{C}$ for $3 \mathrm{sec}$ and $60^{\circ} \mathrm{C}$ for $30 \mathrm{sec}$. The primers (Table III) for collagen $\alpha-2$ (I) chain (COL1A2), leptin (LEP), plasminogen activator inhibitor 1 (SERPINE1) and GAPDH were synthesized by Generay Biotech Co., Ltd.

For the miRNAs, RT-qPCR were performed using the miRNA 1st Strand cDNA Synthesis Kit (Vazyme Biotech Co., Ltd.) and miRNA Universal SYBR qPCR Master Mix (Vazyme Biotech Co., Ltd.), according to the manufacturer's instructions. gDNA was removed by incubating the samples at $42^{\circ} \mathrm{C}$ for $2 \mathrm{~min}$, then cDNA was synthesized at $25^{\circ} \mathrm{C}$ for $5 \mathrm{~min}, 50^{\circ} \mathrm{C}$ for $15 \mathrm{~min}$ and $85^{\circ} \mathrm{C}$ for $5 \mathrm{~min}$. Thermocycling conditions for RT-qPCR consisted of an initial denaturation at $95^{\circ} \mathrm{C}$ for $5 \mathrm{~min}$, followed by 40 cycles at $95^{\circ} \mathrm{C}$ for $10 \mathrm{sec}$ and $60^{\circ} \mathrm{C}$ for $30 \mathrm{sec}$. Specific stem-loop primers and forward 
Table I. Details of the microarrays used.

\begin{tabular}{|c|c|c|c|c|}
\hline $\begin{array}{l}\text { GEO series } \\
\text { number }\end{array}$ & $\begin{array}{l}\text { Healthy } \\
\text { controls, } \mathrm{n}\end{array}$ & $\begin{array}{l}\text { Patients with } \\
\text { EOPE, n }\end{array}$ & Tissue & Platform \\
\hline GSE103542 & 8 & 11 & Placenta & GPL23980, miRLink microRNA Arrays v. 16 \\
\hline GSE74341 & 5 & 7 & Placenta & $\begin{array}{l}\text { GPL16699, Agilent-039494 SurePrint G3 Human } \\
\text { GE v2 8x60K Microarray } 039381 \text { (Feature number version) }\end{array}$ \\
\hline GSE44711 & 8 & 8 & Placenta & GPL10558, Illumina HumanHT-12 v4.0 Expression Beadchip \\
\hline
\end{tabular}

EOPE, early onset preeclampsia; GEO, Gene Expression Omnibus.

Table II. Clinical information of the patients with EOPE $(n=30)$ and NCs $(n=29)$.

\begin{tabular}{lcc}
\hline Clinical characteristics & NC & EOPE \\
\hline Maternal age, years & $28.0 \pm 1.8$ & $27.3 \pm 2.1$ \\
Pregestational BMI, kg/m² & $21.3 \pm 2.1$ & $22.6 \pm 1.3$ \\
SBP, mmHg & $122.5 \pm 5.2$ & $161.0 \pm 3.6^{\mathrm{a}}$ \\
DBP, mmHg & $76.0 \pm 5.1$ & $112.7 \pm 3.2^{\mathrm{a}}$ \\
Proteinuria, mg/24 h & Not tested & $3,065.3 \pm 387.3$ \\
Gestational age at diagnosis, & N/A & $31.0 \pm 1.0$ \\
weeks & & \\
Gestational age at delivery, & $36.0 \pm 1.4$ & $35.0 \pm 1.0$ \\
weeks & & \\
Neonate birth weight, g & $3,007.5 \pm 216.3$ & $2,677.0 \pm 147.5$ \\
\hline P $<0.01$ vs. NC. SBP, systolic blood pressure; DBP, diastolic blood \\
pressure; NC, normal controls; EOPE, early onset preeclampsia.
\end{tabular}

primers for hsa-miR-937, hsa-miR-1486*, hsa-miR-3907 and hsa-miR-367* are listed in Table III. All samples were analysed in triplicate, and gene expression values were normalized to the values of $\beta$-actin using the $2^{-\Delta \Delta \mathrm{Cq}}$ method (33).

Statistical analysis. SPSS 16.0 software (SPSS, Inc.) was used for statistical analysis, and GraphPad Prism 5 software (GraphPad Software, Inc.) was used to produce figures. Quantitative data are presented as the mean \pm SD. For comparisons between two groups, an independent sample t-test was used. $\mathrm{P}<0.05$ was considered to indicate a statistically significant difference.

\section{Results}

Identification of EOPE-associated DEGs. After annotation with official gene symbols, 32,078 genes were found in GSE74341, and 20,929 genes were found in GSE44711. The expression matrixes of GSE74341 and GSE44711 were merged based on their 17,834 common genes. The merged expression matrix was processed with the $\mathrm{R}$ packages RankProd and metaMA separately. A total of 276 genes were outputted by RankProd, and 2,096 were outputted by metaMA; 246 genes were shared between the analyses and identified as DEGs (Fig. 1A). A total of 149 upregulated and 97 downregulated
Table III. Primer sequences.

\begin{tabular}{ll}
\hline Gene & \multicolumn{1}{c}{ Primer sequences $\left(5^{\prime} \rightarrow 3^{\prime}\right)$} \\
\hline COL1A2 & F: GTTGCTGCTTGCAGTAACCTT \\
& R: AGGGCCAAGTCCAACTCCTT \\
LEP & F: TGCCTTCCAGAAACGTGATCC \\
& R: CTCTGTGGAGTAGCCTGAAGC \\
SERPINE1 & F: ACCGCAACGTGGTTTTCTCA \\
RAPDH & R: TTGAATCCCATAGCTGCTTGAAT \\
& F: GGAGCGAGATCCCTCCAAAAT \\
hsa-miR-937-loop & R: GGCTGTTGTCATACTTCTCATGG \\
& GTCGGCAATTGCACTGGATACGAC \\
& GGCAGA
\end{tabular}

hsa-miR-937 F: GGGATCCGCGCTCTGACTC

hsa-miR-148b*-loop GTCGTATCCAGTGCGTGTCGTGGA GTCGGCAATTGCACTGGATACGAC GCCTGA

hsa-miR-148b* F: GGGAAGTTCTGTTATACAC

hsa-miR-3907-loop GTCGTATCCAGTGCGTGTCGTGGA GTCGGCAATTGCACTGGATACGAC TGTGAG

hsa-miR-3907 F: GGGAGGTGCTCCAGGCTGG

hsa-miR-367*-loop GTCGTATCCAGTGCGTGTCGTGGA GTCGGCAATTGCACTGGATACGAC AGAGTT

hsa-miR-367*

Universal reverse F: GGGACTGTTGCTAATATGC primer

U6

F: CGCTTCGGCAGCACATATACTA

R: CGCTTCACGAATTTGCGTGTCA

F, forward; R, reverse; miRNA, microRNA; COL1A2, collagen $\alpha$-2(I) chain; LEP, leptin; SERPINE1, plasminogen activator inhibitor 1.

DEGs were identified in patients with EOPE compared with healthy controls (Fig. 1B). The top 30 upregulated and top 30 downregulated DEGs are shown in Tables IV and V, respectively. To visualize the expression patterns of these DEGs in the EOPE and normal control (NC) groups, clustering heat maps of DEGs were constructed for GSE74341 (Fig. 1C) and GSE44711 (Fig. 1D). 
A

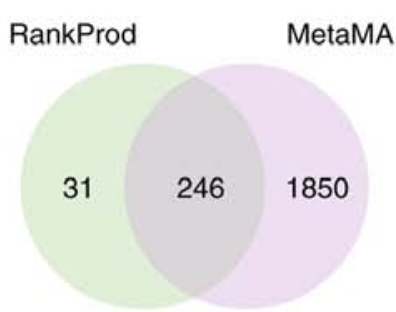

C

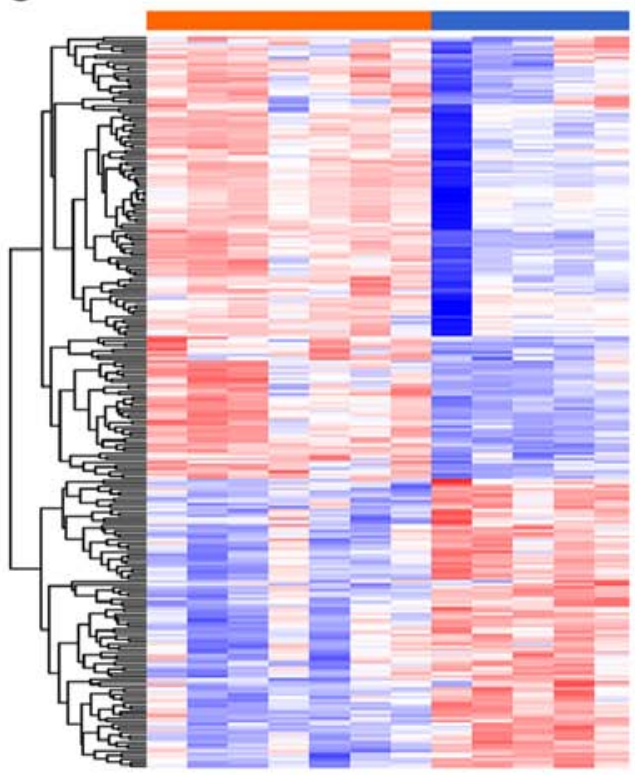

B

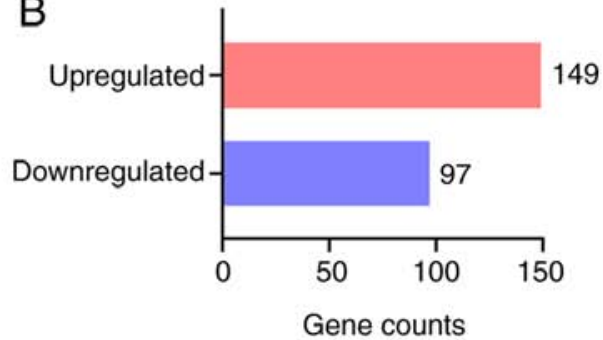

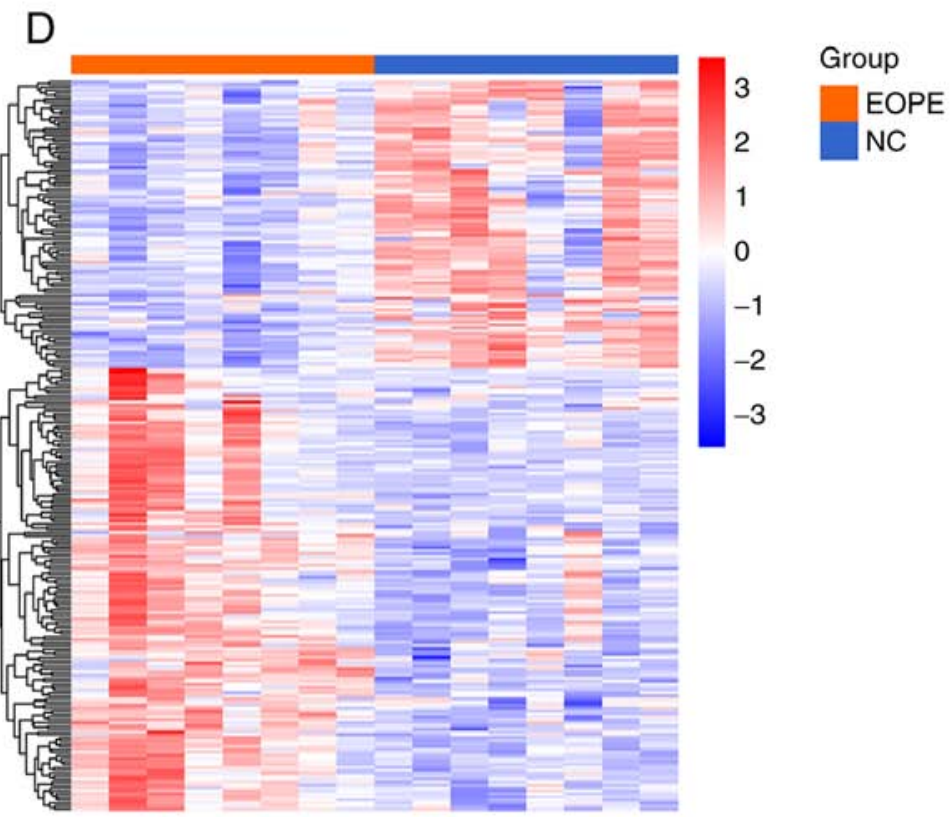

Figure 1. Identification of DEGs between patients with EOPE and healthy controls. (A) A total of 246 DEGs overlapped in the output between the R packages RankProd and metaMA. A total of 277 genes were outputted by RankProd, and 2,096 genes were outputted by metaMA. The 246 shared DEGs were identified by Venn diagram analysis. (B) Number of upregulated and downregulated DEGs. There were 149 upregulated DEGs and 97 downregulated DEGs. Clustering heat maps of DEGs in (C) GSE74341 and (D) GSE44711. The ordinate presents the DEG names, and the abscissa presents the names of the samples. Samples labelled with orange bars are from patients with EOPE, whereas those labelled blue are from healthy controls. Red represents high expression, and blue represents low expression. DEGs, differentially expressed genes; EOPE, early onset preeclampsia; NC, normal control.

Functional annotation of DEGs. To explore the biological functions of the DEGs, the list of DEGs was uploaded to the DAVID database for GO and KEGG enrichment analysis. GO is organized into three ontologies: 'Cellular Component' (CC), 'Molecular Function' (MF) and 'Biological Process' (BP). In the CC ontology (Fig. 2A), DEGs were primarily enriched in the GO terms 'extracellular region', 'extracellular exosome', 'extracellular space' and 'integral component of plasma membrane'. In the MF ontology (Fig. 2B), DEGs were mainly enriched in the terms 'calcium ion binding', 'receptor binding', 'heparin binding' and 'hormone binding'. In the BP ontology (Fig. 2C), DEGs were mainly enriched in the terms 'cell adhesion', 'female pregnancy', 'extracellular matrix organization' and 'response to hypoxia'. KEGG pathway analysis (Fig. 2D) indicated that the DEGs were primarily enriched in the KEGG pathways 'focal adhesion', 'ECM-receptor interaction', 'PI3K-Akt signaling' and 'ovarian steroidogenesis'. The GO and KEGG results suggested that these DEGs were closely related to the occurrence and development of EOPE.

PPI network analysis of DEGs. To explore the interactions among the DEGs, the list of DEGs was uploaded to the STRING database to construct the PPI network. The PPI network contained 174 nodes representing 93 upregulated
DEGs and 81 downregulated DEGs and 488 edges. Significant gene modules of the PPI network were identified using the Cytoscape plugin MCODE, and the first and second ranked modules are shown (Fig. 3A). Degree is a parameter that reflects the number of connected nodes with an individual node. The larger a node's 'Degree value' is, the more likely it is that the node is a hub gene. DEGs were ranked according to their 'Degree values', and top 10 DEGs were considered hub genes (Fig. 3B). The hub genes included epidermal growth factor receptor (EGFR), collagen $\alpha-1(\mathrm{I})$ chain (COL1A1), secreted phosphoprotein 1 (SPP1), LEP, COL1A2, SERPINE1, Thy-1 membrane glycoprotein (THY1), bone morphogenetic protein 4 (BMP4), vascular cell adhesion protein 1 (VCAM1) and matrix metallopeptidase 1 (MMP1).

$\mathrm{GO}$ analysis was performed to explore the BPs associated with module 1, module 2 and hub genes (Fig. 3C). Module 1 was primarily enriched in the GO terms 'extracellular matrix organization', 'positive regulation of $\mathrm{T}$ cell proliferation' and 'negative regulation of blood coagulation'. Module 2 was mainly enriched in the terms 'positive regulation of cell migration', 'extracellular matrix organization' and 'collagen fibril organization'. Hub genes were mainly enriched in the terms 'extracellular matrix organization', 'cellular response to amino acid stimulus' and 'collagen catabolic process'. Of note, 
Table IV. Top 30 upregulated DEGs.

\begin{tabular}{|c|c|c|}
\hline Upregulated genes & $\log 2 \mathrm{FC}$ & pfp \\
\hline $\mathrm{CRH}$ & 4.14 & $1.19 \times 10^{-23}$ \\
\hline HTRA4 & 3.64 & $9.71 \times 10^{-22}$ \\
\hline PSG9 & 3.59 & $4.80 \times 10^{-18}$ \\
\hline EBI3 & 2.94 & $4.73 \times 10^{-18}$ \\
\hline ADAM12 & 2.61 & $3.03 \times 10^{-16}$ \\
\hline LHB & 2.94 & $5.70 \times 10^{-16}$ \\
\hline PRG2 & 2.14 & $1.32 \times 10^{-15}$ \\
\hline PAPPA2 & 3.02 & $2.90 \times 10^{-15}$ \\
\hline SLCO2A1 & 2.50 & $7.08 \times 10^{-15}$ \\
\hline COL17A1 & 2.20 & $1.19 \times 10^{-14}$ \\
\hline LEP & 2.82 & $2.16 \times 10^{-13}$ \\
\hline SIGLEC6 & 2.28 & $3.42 \times 10^{-13}$ \\
\hline PAPPA & 2.22 & $1.14 \times 10^{-12}$ \\
\hline PSG6 & 2.22 & $1.18 \times 10^{-12}$ \\
\hline GDF15 & 2.40 & $2.22 \times 10^{-12}$ \\
\hline GPIHBP1 & 2.14 & $3.93 \times 10^{-12}$ \\
\hline INHA & 2.18 & $3.77 \times 10^{-12}$ \\
\hline PSG5 & 2.15 & $4.34 \times 10^{-12}$ \\
\hline FSTL3 & 2.41 & $4.71 \times 10^{-12}$ \\
\hline CA4 & 1.80 & $1.05 \times 10^{-11}$ \\
\hline PSG1 & 2.18 & $1.15 \times 10^{-11}$ \\
\hline LIMCH1 & 2.05 & $1.21 \times 10^{-11}$ \\
\hline ANKRD37 & 1.99 & $1.51 \times 10^{-11}$ \\
\hline HTRA1 & 2.46 & $1.82 \times 10^{-11}$ \\
\hline PSG3 & 2.17 & $1.90 \times 10^{-11}$ \\
\hline QPCT & 2.13 & $2.81 \times 10^{-11}$ \\
\hline ARMS2 & 2.07 & $4.86 \times 10^{-11}$ \\
\hline PSG11 & 1.91 & $5.98 \times 10^{-11}$ \\
\hline MMP11 & 1.96 & $6.26 \times 10^{-11}$ \\
\hline CYP11A1 & 1.88 & $1.09 \times 10^{-10}$ \\
\hline
\end{tabular}

DEGs, differentially expressed genes; FC, fold-change; pfp, percentage of false prediction.

module 1, module 2 and hub genes were all enriched in the term 'extracellular matrix organization', which indicated that extracellular matrix organization may play important roles in EOPE.

Identification of DEMs and construction of the DEM-target $D E G$ interaction network. The expression matrix of GSE103542 was analysed using the $\mathrm{R}$ package limma, and $\mid \log \mathrm{FCl}>2$ and $\mathrm{P}<0.05$ were applied to define DEMs. A total of 28 miRNAs were differentially expressed between patients with EOPE and healthy controls, among which 20 miRNAs were downregulated and 8 miRNAs were upregulated in patients with EOPE compared with controls (Fig. 4A and Table VI). A clustering heat map of the DEMs was constructed to visualize the differences in the expression patterns of miRNAs between the EOPE and NC groups (Fig. 4B).

The target genes of the DEMs were predicted using miRNAtap, an $\mathrm{R}$ package that integrates sources from the
Table V. Top 30 downregulated DEGs.

\begin{tabular}{lcc}
\hline Downregulated genes & Log2FC & pfp \\
\hline CADM3 & -2.04 & $1.09 \times 10^{-12}$ \\
SPP1 & -1.86 & $1.76 \times 10^{-11}$ \\
BHLHE41 & -1.67 & $4.77 \times 10^{-10}$ \\
SPON1 & -1.68 & $4.86 \times 10^{-10}$ \\
PDPN & -1.55 & $6.06 \times 10^{-10}$ \\
OLFML3 & -1.64 & $6.87 \times 10^{-10}$ \\
CCL13 & -1.56 & $9.12 \times 10^{-10}$ \\
VTN & -1.43 & $1.39 \times 10^{-9}$ \\
ALDH1A1 & -1.50 & $1.70 \times 10^{-9}$ \\
SRPX & -1.54 & $1.66 \times 10^{-9}$ \\
CXCL14 & -1.58 & $2.75 \times 10^{-9}$ \\
DKK1 & -1.28 & $3.67 \times 10^{-9}$ \\
SLC16A10 & -1.43 & $4.04 \times 10^{-9}$ \\
PCOLCE & -1.46 & $4.38 \times 10^{-9}$ \\
CFD & -1.55 & $4.82 \times 10^{-9}$ \\
SLIT2 & -1.53 & $4.81 \times 10^{-9}$ \\
ENPP1 & -1.47 & $1.01 \times 10^{-8}$ \\
CPXM1 & -1.37 & $1.13 \times 10^{-8}$ \\
THY1 & -1.36 & $1.40 \times 10^{-8}$ \\
COL6A2 & -1.34 & $1.57 \times 10^{-8}$ \\
PRRX1 & -1.54 & $2.76 \times 10^{-8}$ \\
METTL7B & -1.34 & $4.18 \times 10^{-8}$ \\
WNT2 & -1.38 & $4.01 \times 10^{-8}$ \\
COL1A1 & -1.37 & $5.74 \times 10^{-8}$ \\
DPT & -1.17 & $6.29 \times 10^{-8}$ \\
CES1 & -1.24 & $7.55 \times 10^{-8}$ \\
SCUBE2 & -1.33 & $1.09 \times 10^{-7}$ \\
FST & -1.18 & $1.83 \times 10^{-7}$ \\
COL6A1 & -1.30 & $2.43 \times 10^{-7}$ \\
GPR34 & -1.21 & $2.36 \times 10^{-7}$ \\
\hline DEGs, differentially & fold-change; pfp, \\
percentage of false prediction. & \\
\hline & & \\
\hline
\end{tabular}

PicTar, DIANA, TargetScan, miranda and mirdb databases to increase the confidence of prediction. The target DEGs were considered DEGs that overlapped with the target genes of DEMs. A DEM-target DEG interaction network was constructed to illustrate the connections between the DEMs and DEGs (Fig. 4C). There were 80 nodes in the network, comprising 59 DEGs (45 upregulated, 14 downregulated) and 21 DEMs (6 upregulated, 15 downregulated). These nodes were connected by 76 edges. The nodes with the largest 'Degree values' were has-miR-19a* and has-miR-30a, which were predicted to regulate the expression of 8 DEGs each.

Local cohort validation of DEM-target DEG patterns. Placenta tissue samples from 30 patients with EOPE and 29 matched healthy controls were collected for RNA extraction. Given that COL1A2, LEP and SERPINE1 were identified as hub genes and predicted to be targets of DEMs, the expression of the DEMs hsa-miR-937, hsa-miR-148b*, hsa-miR-3907 and hsa-miR-367*, and the target DEGs COL1A2, LEP and SERPINE1 were validated using RT-qPCR (Fig. 5). Compared with the corresponding expression in the NC group, the 
A

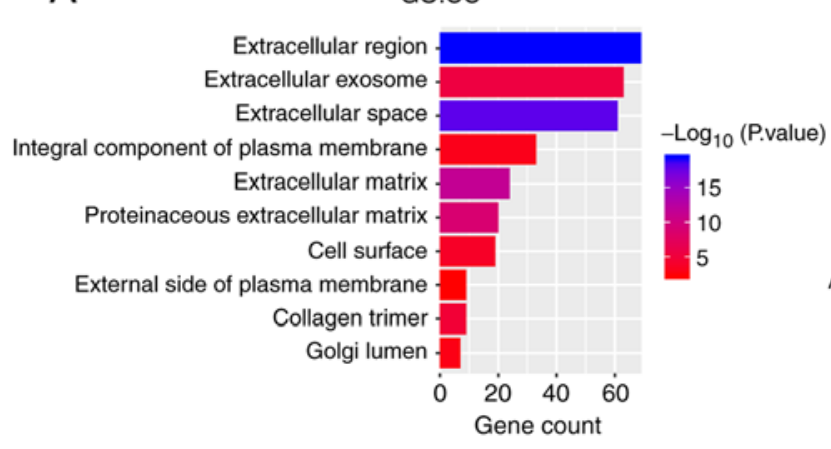

C

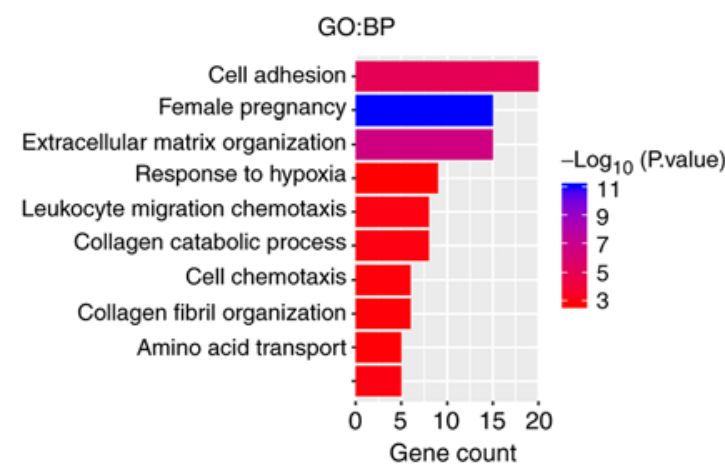

B

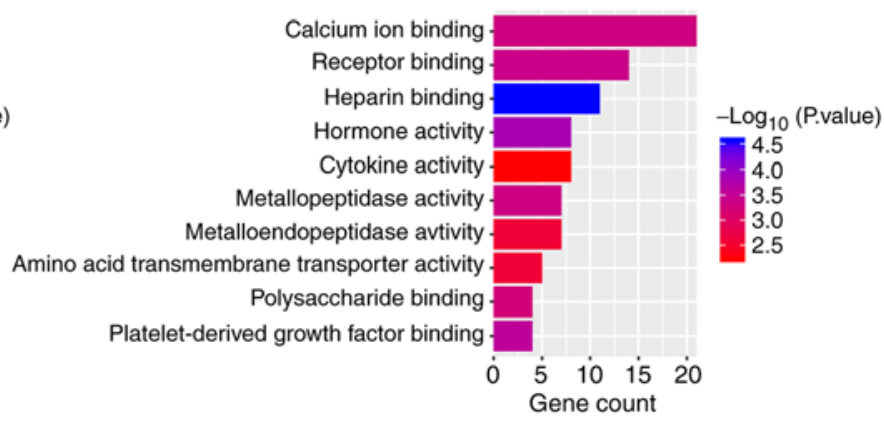

D

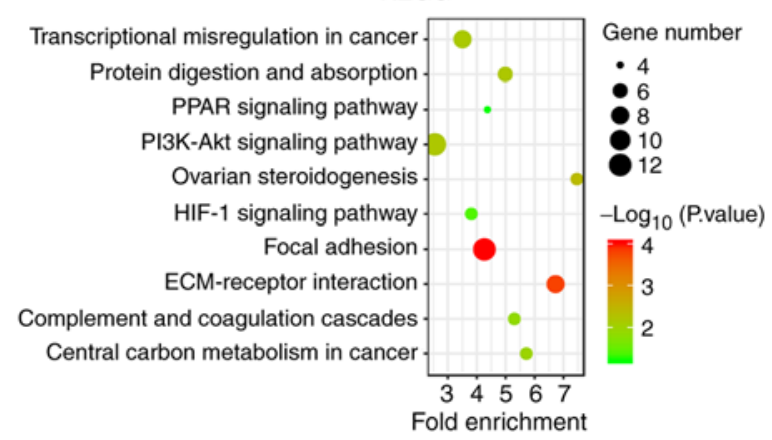

Figure 2. Functional annotation of DEGs. GO analysis of DEGs in the categories of (A) CC, (B) MF and (C) BP. The ordinate displays the GO terms, and the abscissa presents the numbers of DEGs enriched in the GO terms. The-log10 (P-value) is reflected by the colour of the bar. (D) KEGG pathway enrichment of DEGs. The ordinate presents the names of the enriched pathways, and the abscissa presents the fold-enrichment. The number of DEGs enriched in a pathway is denoted by bubble size, and the-log10 (P-value) is reflected by the bubble's colour. DEGs, differentially expressed genes; GO, Gene Ontology; CC, cellular component; MF, molecular function; BP, biological process; KEGG, Kyoto Encyclopedia of Genes and Genomes.

expression levels of hsa-miR-367*, SERPINE1 and LEP were increased significantly in the EOPE group, whereas that of hsa-miR-937, hsa-miR-148b*, hsa-miR-3907 and COL1A2 were decreased significantly in the EOPE group, consistent with the microarray results.

\section{Discussion}

EOPE is a threat to maternal and foetal health worldwide, especially in developing countries (34). The present research employed an integrated approach to investigate alterations of miRNAs and genes in EOPE. In addition, the connections between the altered miRNAs and genes were explored, which may help to explain the pathogenesis of EOPE. In the current study, a total of 246 DEGs and 28 DEMs were identified, among which 59 DEGs and 21 DEMs may have regulatory relationships.

The identified DEGs were primarily associated with BPs, such as 'cell adhesion', 'female pregnancy', 'extracellularmatrix organization' and 'response to hypoxia'. It has been reported that an imbalance of MMPs plays a vital role in the formation of PE (35). In addition, it has been well-established that placenta ischaemia and hypoxia contribute to the development of PE (36). The DEGs were mainly enriched in the following KEGG pathways: 'Focal adhesion', 'ECM-receptor interaction', 'PI3K-Akt signaling' and 'ovarian steroidogenesis'. The PI3K-Akt signalling pathway is a pro-survival pathway that regulates cell proliferation, differentiation and apoptosis (37). PI3K-Akt signalling is typically dysregulated in numerous types of cancer and thus has become an important target for anticancer treatment (38). A role of the PI3K-Akt signalling pathway in PE has also been reported. Cudmore et al (39) proposed that inhibition of the PI3K-Akt signalling pathway increased circulating soluble endoglin release and relieved endothelial dysfunction in PE.

In the present study, using STRING database and Cytoscape, 10 genes (EGFR, COL1A1, SPP1, LEP, COL1A2, SERPINE1, THY1, BMP4, VCAM1 and MMP1) were identified as hub genes that play important roles in EOPE. EGFR signalling has been reported to be overactive in PE and to promote the secretion of soluble FMS-like tyrosine kinase-1, which has been implicated in the pathogenesis of PE (40). The expression of COL1A1 was found to be closely associated with $\mathrm{PE}(\mathrm{P}=0.0011)$ in a large-scale study $(\mathrm{PE}=394$, $\mathrm{NC}=631)$ (41). SPP1 was found to play a role in cytotrophoblast invasion of the maternal vasculature/extracellular matrix during non-preeclamptic placentation (42) and was downregulated in the placenta of patients with PE (43). In the present research, SPP1 was ranked second in the list of downregulated genes, which indicated that SPP1 could serve as biomarker of EOPE and that the role of SPP1 in PE deserves further investigation. LEP in serum has been found to be significantly higher in patients with $\mathrm{PE}$ than in NCs ( $\mathrm{PE}=430, \mathrm{NC}=316)$ (44). Additionally, serum LEP was found to be higher in patients with EOPE than patients with LOPE (45). Genetic variants of SERPINE1 have been found to be associated with PE (46). MMP-1 serves as a mediator of vasoconstriction and vascular dysfunction in PE (47). 


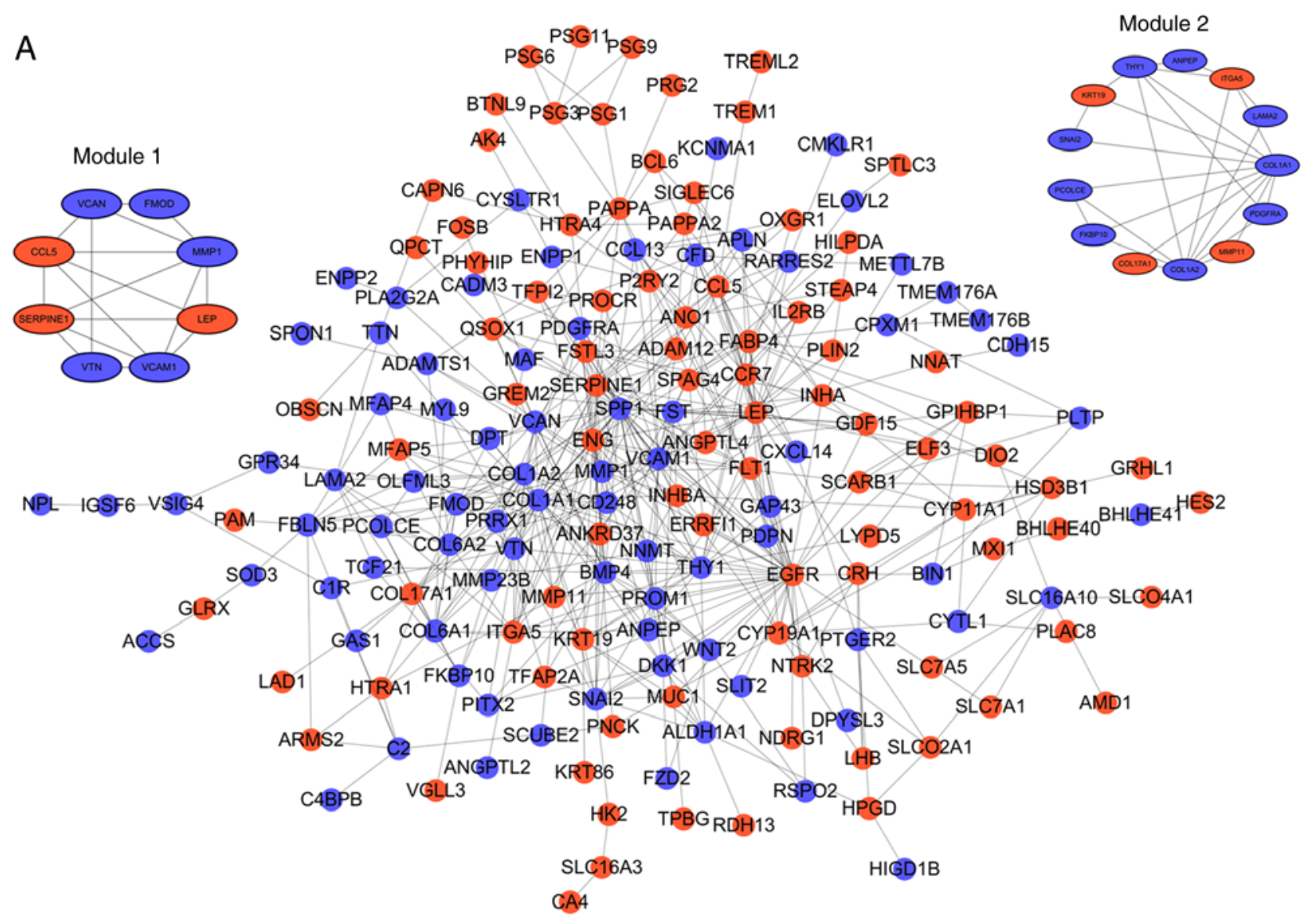

B

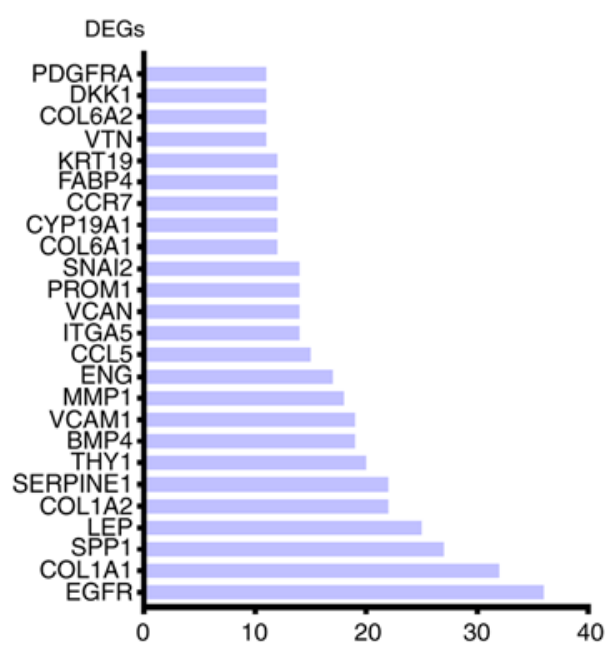

C

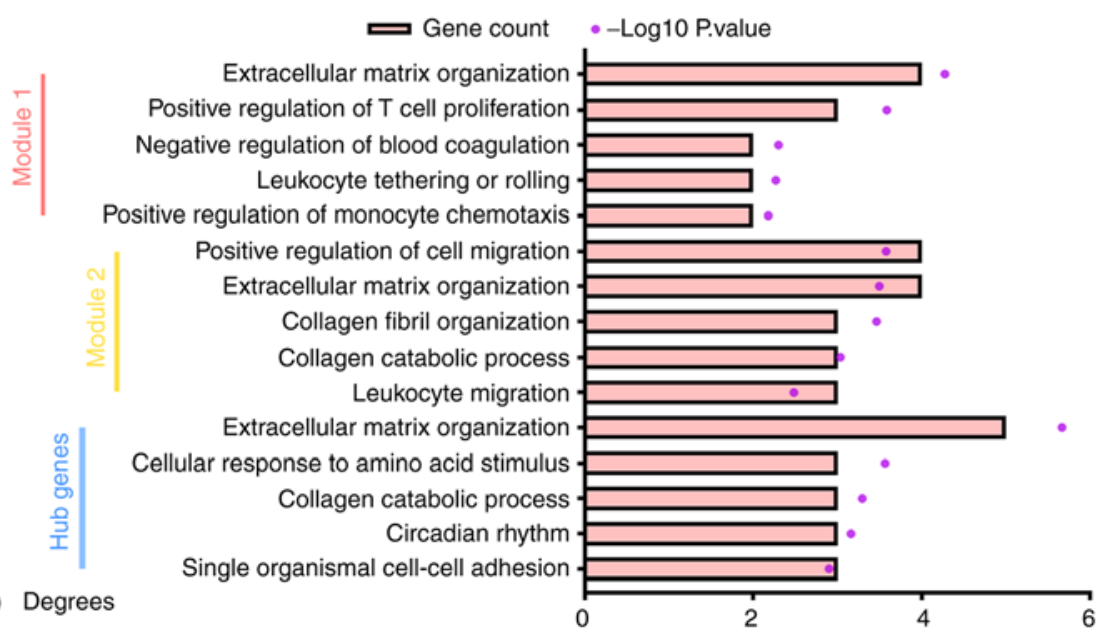

Figure 3. Construction of the PPI network and identification of important modules and hub genes. (A) PPI network of the DEGs and the two most important modules of the network. Nodes of the PPI network represent DEGs, among which red nodes represent upregulated DEGs and blue nodes represent downregulated DEGs. Edges between nodes represent the interactions of proteins encoded by DEGs. (B) Top 25 nodes of the PPI network ranked by Degree value. (C) GO analysis of module 1, module 2 and hub genes. Pink bars represent the number of DEGs enriched in the GO term, and the purple circles represent-log10 (P-value). PPI, Protein-Protein Interaction; DEGs, differentially expressed genes; GO, Gene Ontology.

However, the roles of COL1A2, THY1, BMP4 and VCAM1 in PE remain unclear.

Increasing evidence has indicated that miRNA dysregulation is responsible for the pathogenesis of EOPE. In the present study, 28 placental miRNAs were identified as dysregulated. Previous data demonstrated that hsa-miR-431 inhibited the migration and invasion of trophoblastic cells, which might contribute to the onset of PE (48), whereas hsa-miR-145* played the opposite role (49). Furthermore, Brkić et al (50) revealed that hsa-miR-218 promotes endovascular trophoblast differentiation and spiral artery remodelling, which implies that downregulation of hsa-miR-218 in placenta may contribute to PE development. Additionally, hsa-miR-126* in placenta has been reported to be downregulated in patients with $\mathrm{PE}$, which 


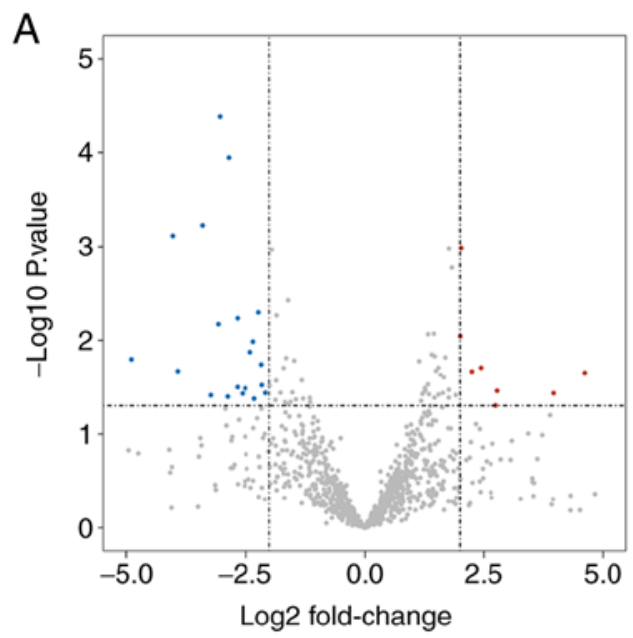

B

C
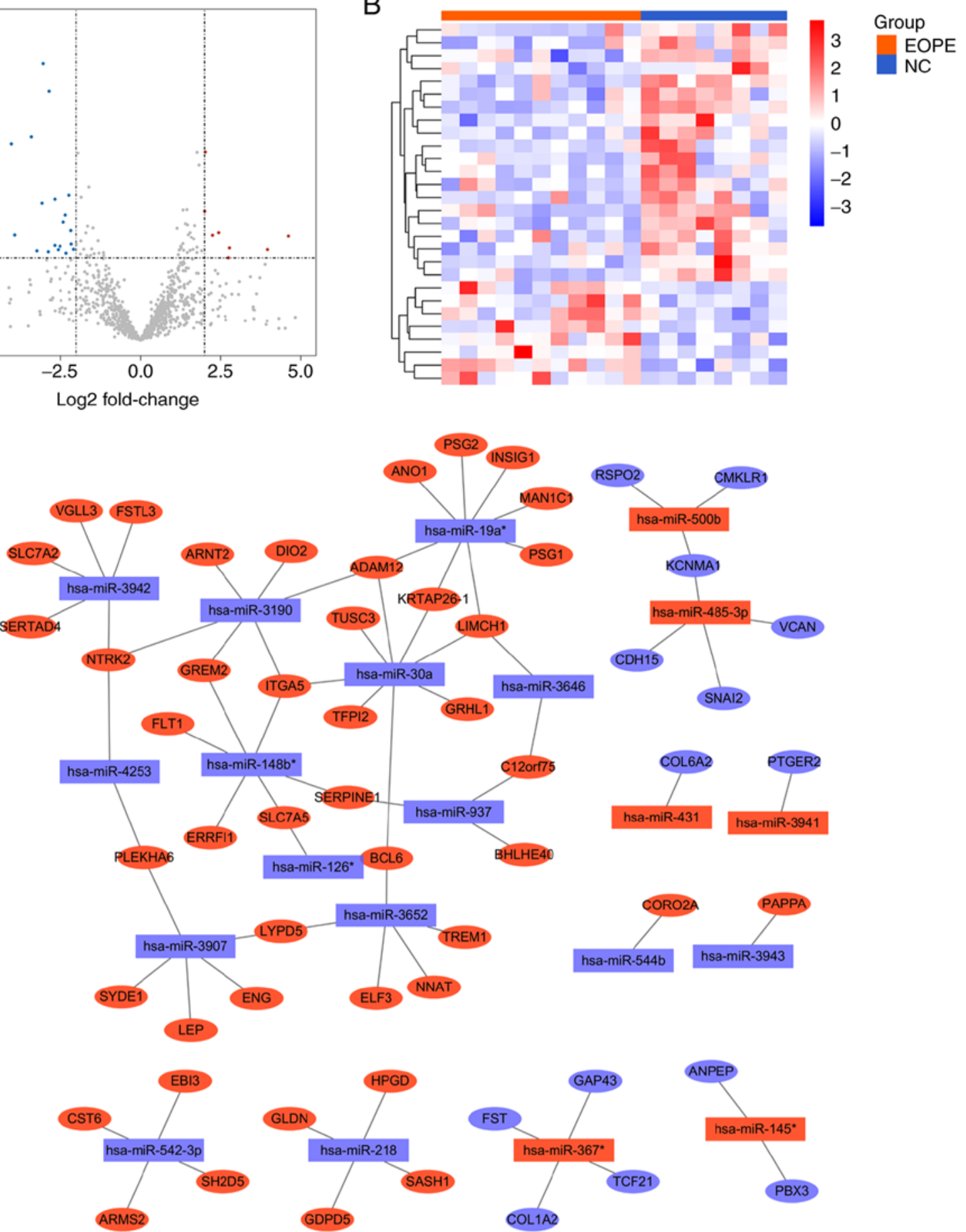

Figure 4. Identification of DEMs and construction of the DEM-target DEG interaction network. (A) A volcano plot of miRNAs in GSE103542, with criteria of $\mathrm{P}<0.05$ and $\log 2 \mathrm{FCl}=2$ applied to identify DEMs. (B) Clustering heat map of DEMs in GSE103542. The ordinate displays the names of the DEMs, and the abscissa presents the sample names. Samples labelled with orange bars are from patients with EOPE, whereas those labelled blue are from healthy controls. Red represents high expression, and blue represents low expression. (C) Interaction network of DEMs and their target DEGs. Rectangular nodes represent DEMs, and oval nodes represent DEGs. Red nodes represent upregulated DEMs, and blue nodes represent downregulated DEMs. Edges between nodes represent interactions between DEMs and DEGs. miRNA, microRNA; FC, fold-change; DEMs, differentially expressed miRNAs; EOPE, early onset preeclampsia; $\mathrm{NC}$, normal control; DEGs, differentially expressed genes.

is consistent with the microarray results of the present study, and the expression of hsa-miR-126* has been identified as correlated with vascular endothelial growth factor levels (51). Furthermore, a previous study indicated that hsa-miR-126* is essential for the angiogenic properties of endothelial progenitor cells in vitro and for placental vasculogenesis in vivo (52). In the current study, four DEM-hub gene pairs were predicted.
Using local samples, the expression changes of hsa-miR-937, hsa-miR-148b*, hsa-miR-3907, hsa-miR-367*, COL1A2, LEP and SERPINE1 were validated in the placenta of patients with PE compared with controls.

With the rapid development of high-throughput technologies, numerous studies have been performed to investigate the molecular mechanisms of EOPE by examining transcriptional 
Table VI. List of DEMs.

\begin{tabular}{lll}
\hline DEMs & logFC & P-value \\
\hline hsa-miR-1914 & 2.03 & $1.03 \times 10^{-3}$ \\
hsa-miR-431 & 2.01 & $9.02 \times 10^{-3}$ \\
hsa-miR-485-3p & 2.44 & $1.95 \times 10^{-2}$ \\
hsa-miR-500b & 2.25 & $2.15 \times 10^{-2}$ \\
hsa-miR-145* & 4.62 & $2.21 \times 10^{-2}$ \\
hsa-miR-3941 & 2.79 & $3.46 \times 10^{-2}$ \\
hsa-miR-367* & 3.97 & $3.67 \times 10^{-2}$ \\
hsa-miR-875-3p & 2.76 & $4.95 \times 10^{-2}$ \\
hsa-miR-542-3p & -3.04 & $4.07 \times 10^{-5}$ \\
hsa-miR-126* & -2.85 & $1.13 \times 10^{-4}$ \\
hsa-miR-544b & -3.40 & $5.93 \times 10^{-4}$ \\
hsa-miR-3652 & -4.02 & $7.67 \times 10^{-4}$ \\
hsa-miR-2276 & -2.22 & $5.02 \times 10^{-3}$ \\
hsa-miR-937 & -2.67 & $5.80 \times 10^{-3}$ \\
hsa-miR-3907 & -3.07 & $6.70 \times 10^{-3}$ \\
hsa-miR-3190 & -2.33 & $1.03 \times 10^{-2}$ \\
hsa-miR-4253 & -2.40 & $1.33 \times 10^{-2}$ \\
hsa-miR-1274a & -4.89 & $1.59 \times 10^{-2}$ \\
hsa-miR-3942 & -2.16 & $1.80 \times 10^{-2}$ \\
hsa-miR-1471 & -3.92 & $2.13 \times 10^{-2}$ \\
hsa-miR-148b* & -2.15 & $3.01 \times 10^{-2}$ \\
hsa-miR-218 & -2.67 & $3.16 \times 10^{-2}$ \\
hsa-miR-1537 & -2.51 & $3.26 \times 10^{-2}$ \\
hsa-miR-3943 & -2.07 & $3.64 \times 10^{-2}$ \\
hsa-miR-19a* & -2.56 & $3.85 \times 10^{-2}$ \\
hsa-miR-3646 & -3.23 & $19 \times 10^{-2}$ \\
hsa-miR-302a & -2.88 & \\
hsa-miR-30a & -2.31 & \\
\hline & & \\
\hline
\end{tabular}

DEMs, differentially expressed miRNAs; FC, fold-change; miR/miRNA, microRNA.

changes. He et al (53), Ma et al (54) and Song et al (55) analysed microarray data from GSE44711 to identify candidate markers and pathways in EOPE. Owing to the different bioinformatics approaches among the studies, the results of the three studies varied. Gunel et al (56) and Betoni et al (57) investigated miRNA profiles in the placentas of patients with PE without distinguishing EOPE from LOPE, whereas Lykoudi et al (58) analysed dysregulated placental miRNAs in patients with EOPE. However, the aforementioned studies did not explore the regulatory relationships between mRNAs and miRNAs; such relationships may contribute to the pathogenesis of EOPE. Yang-Dong et al (59) constructed a miRNA-mRNA regulatory network for EOPE by analysing microarray data from GSE103542 and GSE74341. In contrast to this previous study, the present study analysed two microarrays comprehensively to identify DEGs, thus increasing the strength and validity of the results of this study. Furthermore, in the present study, hub genes among the DEGs were identified and partially validated using RT-qPCR, which may provide insight into the molecular mechanisms of EOPE. Nevertheless, there are a few limitations

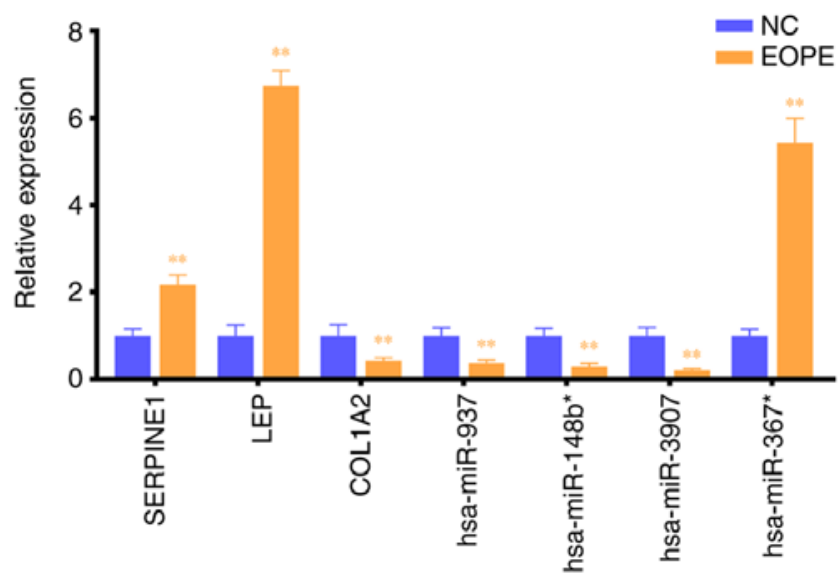

Figure 5. Local cohort validation. Expression of hsa-miR-937, hsa-miR-148b*, hsa-miR-3907, hsa-miR-367*, COL1A2, LEP and SERPINE1 in placenta tissues from NC and EOPE groups as determined via RT-qPCR. Expression levels of GAPDH and U6 snoRNA were used as internal references. ${ }^{* *} \mathrm{P}<0.01$ vs. NC. miRNA, microRNA; COL1A2, collagen $\alpha-2(\mathrm{I})$ chain; LEP, leptin; SERPINE1, plasminogen activator inhibitor 1; NC, normal controls; EOPE, early onset preeclampsia; RT-qPCR, reverse transcription-quantitative polymerase chain reaction.

of the current study. This study failed to demonstrate the exact functions of these hub genes and the associated mechanisms, an important topic that requires further research.

The present research identified DEGs and DEMs associated with EOPE, and provided insight into the relationships between them. EGFR, COL1A1, SPP1, LEP, COL1A2, SERPINE1, THY1, BMP4, VCAM1 and MMP1 could serve as potential biomarkers of EOPE and treatment targets. Furthermore, hsa-miR-937, hsa-miR-148b*, hsa-miR-3907 and hsa-miR-367* could serve as regulators of COL1A2, LEP and SERPINE1, a possibility that warrants further investigation.

\section{Acknowledgements}

Not applicable.

\section{Funding}

The present study was supported by the National Science Foundation of China (grant nos. 81771604, 81601300 and 81901490).

\section{Availability of data and materials}

All data generated or analyzed during this study are included in this published article.

\section{Authors' contributions}

HZ, LX, ZM and HD designed the present study, which was performed by HZ, LX YL and XY. LX, YL and XY made substantial contributions to acquisition and analysis of data. $\mathrm{HZ}$ and $\mathrm{YZ}$ also made contributions to interpretation of data. LX wrote the initial draft of the manuscript. HZ revised it critically for important intellectual content. ZM and HD gave final approval of the version to be published. All authors read and approved the final manuscript. 


\section{Ethics approval and consent to participate}

This research was approved by The Human Research Ethics Committee of Women's Hospital of Nanjing Medical University (approval no. KY-024). All women participating in the study signed informed consent for the collection of placenta tissues and clinical information.

\section{Patient consent for publication}

Not applicable.

\section{Competing interests}

The authors declare that they have no competing interests.

\section{References}

1. Steegers EA, von Dadelszen P, Duvekot JJ and Pijnenborg R: Pre-eclampsia. Lancet 376: 631-644, 2010.

2. Ghulmiyyah L and Sibai B: Maternal mortality from preeclampsia/eclampsia. Semin Perinatol 36: 56-59, 2012.

3. Clark SL, Belfort MA, Dildy GA, Herbst MA, Meyers JA and Hankins GD: Maternal death in the 21st century: Causes, prevention, and relationship to cesarean delivery. Am J Obstet Gynecol 199: 36.e1-e5, 91-2. e7-e11, 2008

4. Roberts JM and Cooper DW: Pathogenesis and genetics of pre-eclampsia. Lancet 357: 53-56, 2001

5. von Dadelszen P, Magee LA and Roberts JM: Subclassification of preeclampsia. Hypertens Pregnancy 22: 143-148, 2003.

6. Lisonkova S, Sabr Y, Mayer C, Young C, Skoll A and Joseph KS Maternal morbidity associated with early-onset and late-onset preeclampsia. Obstet Gynecol 124: 771-781, 2014.

7. Brosens I, Pijnenborg R, Vercruysse L and Romero R: The 'Great Obstetrical Syndromes' are associated with disorders of deep placentation. Am J Obstet Gynecol 204: 193-201, 2011

8. Rupaimoole R and Slack FJ: MicroRNA therapeutics: Towards a new era for the management of cancer and other diseases. Nat Rev Drug Discov 16: 203-222, 2017.

9. Hata A: Functions of microRNAs in cardiovascular biology and disease. Annu Rev Physiol 75: 69-93, 2013.

10. Wang Y, Jiao J, Ren P and Wu M: Upregulation of miRNA-223-3p ameliorates RIP3-mediated necroptosis and inflammatory responses via targeting RIP3 after spinal cord injury. J Cell Biochem: Feb 28 , 2019 (Epub ahead of print).

11. Ranz JM and Machado CA: Uncovering evolutionary patterns of gene expression using microarrays. Trends Ecol Evol 21: 29-37, 2006.

12. Huang GH, Cao XY, Li YY, Zhou CC, Li L, Wang K, Li H, Yu P, Jin Y and Gao L: Gene expression profile of the hippocampus of rats subjected to traumatic brain injury. J Cell Biochem 120: $15776-15789,2019$

13. Tan J, Hu L, Yang X, Zhang X, Wei C, Lu Q, Chen Z and Li J: miRNA expression profiling uncovers a role of miR-302b-3p in regulating skin fibroblasts senescence. J Cell Biochem 121: 70-80, 2020.

14. Liu Z, Xie M, Yao Z, Niu Y, Bu Y and Gao C: Three meta-analyses define a set of commonly overexpressed genes from microarray datasets on astrocytomas. Mol Neurobiol 47: 325-336, 2013.

15. Barrett T, Wilhite SE, Ledoux P, Evangelista C, Kim IF, Tomashevsky M, Marshall KA, Phillippy KH, Sherman PM, Holko M, et al: NCBI GEO: Archive for functional genomics data sets-update. Nucleic Acids Res 41: D991-D995, 2013.

16. Del Carratore F, Jankevics A, Eisinga R, Heskes T, Hong F and Breitling R: RankProd 2.0: A refactored bioconductor package for detecting differentially expressed features in molecular profiling datasets. Bioinformatics 33: 2774-2775, 2017.

17. Marot G, Foulley JL, Mayer CD and Jaffrézic F: Moderated effect size and P-value combinations for microarray meta-analyses. Bioinformatics 25: 2692-2699, 2009.

18. Heberle H, Meirelles GV, da Silva FR, Telles GP and Minghim R InteractiVenn: A web-based tool for the analysis of sets through Venn diagrams. BMC Bioinformatics 16: 169, 2015.
19. Kolde R: Pheatmap: Pretty heatmaps. R package version 1 , 2012.

20. Huang da W, Sherman BT and Lempicki RA: Systematic and integrative analysis of large gene lists using DAVID bioinformatics resources. Nat Protoc 4: 44-57, 2009.

21. Huang da W, Sherman BT and Lempicki RA: Bioinformatics enrichment tools: Paths toward the comprehensive functional analysis of large gene lists. Nucleic Acids Res 37: 1-13, 2009.

22. The Gene Ontology Consortium: The gene ontology resource: 20 Years and still GOing strong. Nucleic Acids Res 47: D330-D338, 2019.

23. Ashburner M, Ball CA, Blake JA, Botstein D, Butler H, Cherry JM, Davis AP, Dolinski K, Dwight SS, Eppig JT, et al: Gene ontology: Tool for the unification of biology. The gene ontology consortium. Nat Genet 25: 25-29, 2000.

24. Kanehisa M and Goto S: KEGG: Kyoto encyclopedia of genes and genomes. Nucleic Acids Res 28: 27-30, 2000.

25. Szklarczyk D, Franceschini A, Wyder S, Forslund K, Heller D, Huerta-Cepas J, Simonovic M, Roth A, Santos A, Tsafou KP, et al: STRING v10: Protein-protein interaction networks, integrated over the tree of life. Nucleic Acids Res 43: D447-D452, 2015.

26. Shannon P, Markiel A, Ozier O, Baliga NS, Wang JT, Ramage D, Amin N, Schwikowski B and Ideker T: Cytoscape: A software environment for integrated models of biomolecular interaction networks. Genome Res 13: 2498-2504, 2003.

27. Bader GD and Hogue CW: An automated method for finding molecular complexes in large protein interaction networks. BMC Bioinformatics 4: 2, 2003.

28. Ritchie ME, Phipson B, Wu D, Hu Y, Law CW, Shi W and Smyth GK: limma powers differential expression analyses for RNA-sequencing and microarray studies. Nucleic Acids Res 43: e47, 2015

29. Wickham H: ggplot2: Elegant graphics for data analysis. Springer International Publishing, 2016.

30. Pajak M and Simpson TI: miRNAtap: miRNAtap: microRNA Targets-Aggregated Predictions. R package version 1.22.0, 2020.

31. Dhawan A, Scott JG, Harris AL and Buffa FM: Pan-cancer characterisation of microRNA across cancer hallmarks reveals microRNA-mediated downregulation of tumour suppressors. Nat Commun 9: 5228, 2018

32. Uzan J, Carbonnel M, Piconne O, Asmar R and Ayoubi JM: Pre-eclampsia: Pathophysiology, diagnosis, and management. Vasc Health Risk Manag 7: 467-474, 2011.

33. Livak KJ and Schmittgen TD: Analysis of relative gene expression data using real-time quantitative PCR and the 2(-Delta Delta C(T)) method. Methods 25: 402-408, 2001.

34. Osungbade KO and Ige OK: Public health perspectives of preeclampsia in developing countries: Implication for health system strengthening. J Pregnancy 2011: 481095, 2011.

35. Chen J and Khalil RA: Matrix metalloproteinases in normal pregnancy and preeclampsia. Prog Mol Biol Transl Sci 148: 87-165, 2017.

36. KarumanchiSA andBdolah Y:Hypoxia and sFlt-1 in preeclampsia: The 'Chicken-and-Egg' question. Endocrinology 145: 4835-4837, 2004.

37. Janku F, Yap TA and Meric-Bernstam F: Targeting the PI3K pathway in cancer: Are we making headway? Nat Rev Clin Oncol 15: 273-291, 2018.

38. Mayer IA and Arteaga CL: The PI3K/AKT pathway as a target for cancer treatment. Annu Rev Med 67: 11-28, 2016

39. Cudmore MJ, Ahmad S, Sissaoui S, Ramma W, Ma B, Fujisawa T, Al-Ani B, Wang K, Cai M, Crispi F, et al: Loss of Akt activity increases circulating soluble endoglin release in preeclampsia: Identification of inter-dependency between Akt-1 and heme oxygenase-1. Eur Heart J 33: 1150-1158, 2012.

40. Hastie R, Brownfoot FC, Pritchard N, Hannan NJ, Cannon P, Nguyen V, Palmer K, Beard S, Tong S and Kaitu'u-Lino TJ: EGFR (Epidermal Growth Factor Receptor) signaling and the mitochondria regulate sFlt-1 (Soluble FMS-Like Tyrosine Kinase-1) secretion. Hypertension 73: 659-670, 2019.

41. Goddard KA, Tromp G, Romero R, Olson JM, Lu Q, Xu Z, Parimi N, Nien JK, Gomez R, Behnke E, et al: Candidate-gene association study of mothers with pre-eclampsia, and their infants, analyzing 775 SNPs in 190 genes. Hum Hered 63: 1-16, 2007.

42. Gabinskaya T, Salafia CM, Gulle VE, Holzman IR and Weintraub AS: Gestational age-dependent extravillous cytotrophoblast osteopontin immunolocalization differentiates between normal and preeclamptic pregnancies. Am J Reprod Immunol 40: 339-346, 1998 
43. Xia J, Qiao F, Su F and Liu H: Implication of expression of osteopontin and its receptor integrin alphanubeta3 in the placenta in the development of preeclampsia. J Huazhong Univ Sci Technolog Med Sci 29: 755-760, 2009.

44. Taylor BD, Ness RB, Olsen J, Hougaard DM, Skogstrand K, Roberts JM and Haggerty CL: Serum leptin measured in early pregnancy is higher in women with preeclampsia compared with normotensive pregnant women. Hypertension 65: 594-599, 2015.

45. Salimi S, Farajian-Mashhadi F, Naghavi A, Naghavi A Mokhtari M, Shahrakipour M, Saravani M and Yaghmaei M: Different profile of serum leptin between early onset and late onset preeclampsia. Dis Markers 2014: 628476, 2014.

46. Buurma AJ, Turner RJ, Driessen JH, Mooyaart AL, Schoones JW, Bruijn JA, Bloemenkamp KW, Dekkers OM and Baelde HJ Genetic variants in pre-eclampsia: A meta-analysis. Hum Reprod Update 19: 289-303, 2013.

47. Estrada-Gutierrez G, Cappello RE, Mishra N, Romero R, Strauss JF III and Walsh SW: Increased expression of matrix metalloproteinase-1 in systemic vessels of preeclamptic women: A critical mediator of vascular dysfunction. Am J Pathol 178: 451-460, 2011.

48. Yang X and Meng T: MicroRNA-431 affects trophoblast migration and invasion by targeting ZEB1 in preeclampsia. Gene 683 : 225-232, 2019.

49. Lv Y, Lu X, Li C, Fan Y, Ji X, Long W, Meng L, Wu L, Wang L, Lv $\mathrm{M}$ and Ding H: miR-145-5p promotes trophoblast cell growth and invasion by targeting FLT1. Life Sci 239: 117008, 2019.

50. Brkić J, Dunk C, O'Brien J,Fu G, Nadeem L, Wang YL, Rosman D, Salem M, Shynlova O, Yougbaré I, et al: MicroRNA-218-5p promotes endovascular trophoblast differentiation and spiral artery remodeling. Mol Ther 26: 2189-2205, 2018.

51. Hong F, Li Y and Xu Y: Decreased placental miR-126 expression and vascular endothelial growth factor levels in patients with pre-eclampsia. J Int Med Res 42: 1243-1251, 2014.
52. Yan T, Liu Y, Cui K, Hu B, Wang F and Zou L: MicroRNA-126 regulates EPCs function: Implications for a role of miR-126 in preeclampsia. J Cell Biochem 114: 2148-2159, 2013.

53. He P, Shao D, Ye M and Zhang G: Analysis of gene expression identifies candidate markers and pathways in pre-eclampsia. J Obstet Gynaecol 35: 578-584, 2015.

54. Ma Y, Lin H, Zhang H, Song X and Yang H: Identification of potential crucial genes associated with early-onset pre-eclampsia via a microarray analysis. J Obstet Gynaecol Res 43: 812-819, 2017.

55. Song J, Li Y and An RF: Identification of early-onset preeclampsia-related genes and MicroRNAs by bioinformatics approaches. Reprod Sci 22: 954-963, 2015.

56. Gunel T, Hosseini MK, Gumusoglu E, Kisakesen HI, Benian A and Aydinli K: Expression profiling of maternal plasma and placenta microRNAs in preeclamptic pregnancies by microarray technology. Placenta 52: 77-85, 2017.

57. Betoni JS, Derr K, Pahl MC, Rogers L, Muller CL, Packard RE, Carey DJ, Kuivaniemi H and Tromp G: MicroRNA analysis in placentas from patients with preeclampsia: Comparison of new and published results. Hypertens Pregnancy 32: 321-339, 2013.

58. Lykoudi A, Kolialexi A, Lambrou GI, Braoudaki M, Siristatidis C, Papaioanou GK, Tzetis M, Mavrou A and Papantoniou N: Dysregulated placental microRNAs in early and late onset preeclampsia. Placenta 61: 24-32, 2018.

59. Yang-Dong O, Ya-Xian L and Xue-qiong Z: Excavation and bioinformatics analysis of early-onset preeclampsia related MicroRNA and target genes. J Nongken Med 40: 494-499, 2018.

This work is licensed under a Creative Commons Attribution-NonCommercial-NoDerivatives 4.0 International (CC BY-NC-ND 4.0) License. 